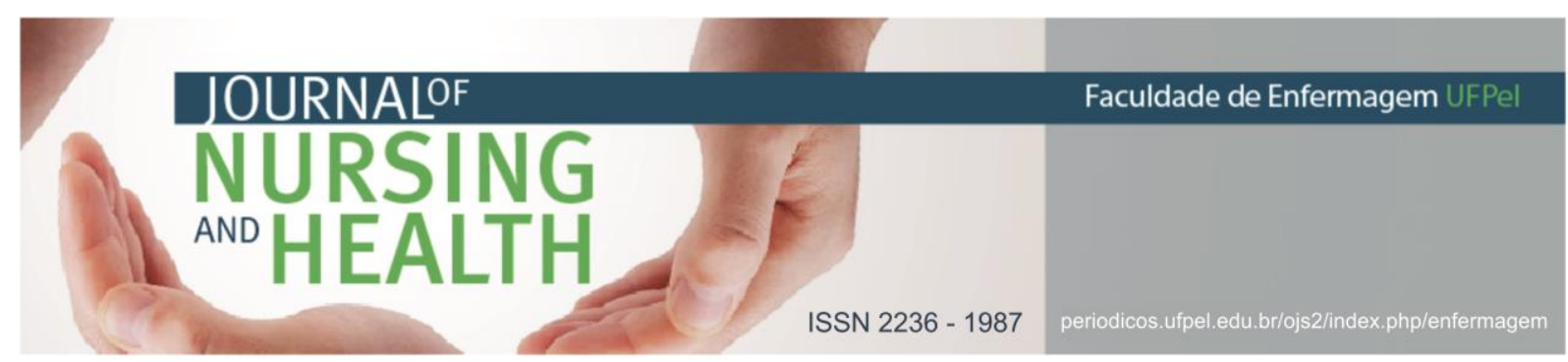

ARTIGO DE REVISÃO

\title{
Intercâmbio internacional nos cursos de graduação de enfermagem
}

\author{
International exchange in nursing undergraduate courses
}

\author{
Intercambio internacional en cursos de grado de enfermería
}

\author{
Guedes, Glauteice Freitas ${ }^{1}$; Silva, Gilberto Tadeu Reis²; Amestoy, Simone Coelho ${ }^{3}$; Silva, Rosana
} Maria de Oliveira ${ }^{4}$; Silva, Cristiane Costa Reis ${ }^{5}$

\begin{abstract}
RESUMO
Objetivo: analisar a produção científica sobre intercâmbios internacionais realizados por estudantes durante a graduação em Enfermagem. Método: trata-se de uma revisão integrativa realizada nas bases de dados Literatura Latino-americana e do Caribe em Ciências da Saúde, Medical Literature Analysis and Retrieval System Online e Cochrane Library no período de 1995 a 2015. Resultados: foram analisados 19 artigos, a maioria de natureza qualitativa, publicados nos Estados Unidos da América, Austrália e Brasil. Destaca-se que o intercâmbio possibilita o desenvolvimento profissional e pessoal do estudante. 0 idioma e choque cultural emergiram como desafios e barreiras. Quanto às atividades desenvolvidas, foram identificadas consultas de enfermagem, estágios em hospitais, visitas técnicas, participação em grupo de pesquisas e eventos científicos. Conclusões: os intercâmbios no campo da Enfermagem ainda se encontram incipientes, entretanto, trata-se de um fenômeno em expansão por estar atrelado aos efeitos da globalização e que precisam de mais investimentos governamentais e privados.

Descritores: Intercâmbio educacional internacional; Internacionalidade; Educação em enfermagem; Enfermagem.
\end{abstract}

\section{ABSTRACT}

Objective: to analyze the scientific production on international exchanges experienced by students during undergraduate nursing. Method: it is an integrative review of the literature performed by searching in the Latin American and Caribbean Literature in Health Sciences, Medical Literature Analysis and Retrieval System Online and Cochrane Library databases from 1995 to 2015. Results: it analyzed 19 articles, most of qualitative studies, published in the United States of America, Australia and Brazil. It is emphasized that the exchange allows the professional and personal development of the student. Language and cultural shock have emerged as challenges and barriers. Regarding the activities developed, nursing appointments, hospital internships, technical visits, participation in a

\footnotetext{
${ }^{1}$ Enfermeira. Mestre em Ciências da Saúde. Professora da Universidade Anhembi Morumbi, São Paulo, SP, Brasil. E-mail: teice1976@hotmail.com

2 Enfermeiro. Pós-doutor em Ensino em Ciências da Saúde. Professor Titular na Universidade Federal da Bahia, Salvador, Bahia, Brasil. E-mail: gtadeuccreis@uol.com.br

${ }^{3}$ Enfermeira. Doutora em Enfermagem. Professora Adjunta na Universidade Federal da Bahia, Salvador, Bahia, Brasil. E-mail: simoneamestoy@hotmail.com

${ }^{4}$ Enfermeira. Doutora em Enfermagem. Professora Adjunta na Universidade Federal da Bahia, Salvador, Bahia, Brasil. E-mail: rosanaosilva@hotmail.com

${ }^{5}$ Enfermeira. Doutora em Ciências da Saúde. Professora na Universidade Salvador, Salvador, Bahia, Brasil. Email: cristianereisfb@gmail.com
} 


\section{JOURNALOF

research group and scientific events were identified. Conclusions: nursing exchanges are still incipient, but it is a phenomenon that is expanding because it is linked to the effects of globalization and requires more government and private investments.

Descriptors: International educational exchange; Internationality; Education, Nursing. Nursing.

\section{RESUMEN}

Objetivo: analizar la producción científica sobre intercambios internacionales realizados por estudiantes durante la graduación en Enfermería. Método: revisión integrativa realizada en las bases de datos LILACS, Medical Literature Analysis and Retrieval System Online y Cochrane Library en el período de 1995 a 2015. Resultados: se analizaron 19 artículos, la mayoría cualitativo, publicados en los Estados Unidos de América, Australia y Brasil. Se destaca que el intercambio posibilita el desarrollo profesional y personal del estudiante. El lenguaje y el choque cultural surgieron como desafíos y barreras. En cuanto a las actividades desarrolladas se identificaron consultas de enfermería, pasantías en hospitales, visitas técnicas, participación en grupo de investigaciones y eventos científicos. Conclusiones: los intercambios en el campo de la enfermería todavía se encuentran incipientes, sin embargo, se trata de un fenómeno en expansión por estar ligado a los efectos de la globalización y que necesitan más inversiones gubernamentales y privadas.

Descriptores: Intercambio educacional internacional; Internacionalidad; Educación en enfermería; Enfermería.

\section{INTRODUÇÃO}

A realização de intercâmbio educacional internacional durante a graduação é uma tendência, cuja origem está atrelada aos efeitos da globalização, sobretudo, em países emergentes como o Brasil. 0 aumento na competitividade junto ao crescimento tecnológico são exemplos de mudanças nesses países, pois as privatizações e a introdução de novos padrões internacionais impactam diversos setores da economia brasileira, principalmente na indústria, nos serviços, na gestão pública e na educação. ${ }^{1}$

Acresce-se que as relações de trabalho, também decorrentes das mudanças estruturais impostas pelo capitalismo mundial, desenham novos cenários nos espaços de produção e serviço por meio da criação e implantação de inovadoras tecnologias (máquinas, processos), tornando-os mais competitivos e produtivos. Trata- se de um contexto que exige qualificação dos trabalhadores, perfis profissionais multicompetentes e produção de conhecimentos, elementos historicamente essenciais à competividade. Dessa forma, a globalização impulsiona níveis mais altos de exigência da formação profissional. ${ }^{2-3}$

Nota-se, portanto, que os efeitos da globalização atingem diretamente o mercado de trabalho mundial, fato que, por sua vez, obriga as instituições de ensino a iniciarem um processo de adaptação às novas exigências profissionais. Assim, entre as várias estratégias adotadas pelos órgãos legisladores das políticas públicas de saúde e educação, destaca-se a busca da internacionalização do ensino superior em diversas universidades brasileiras.

As ações de implementação do intercâmbio educacional estão 


\section{JOURNAIOF \\ NURSING \\ MNHEALTH}

voltadas para proporcionar ao estudante interações com pessoas de distintas origens e culturas no universo acadêmico, levando-o a conhecer diferentes visões de mundo, ampliando horizontes e perspectivas profissionais, além de dar visibilidade às suas instituições de origem. Essas experiências favorecem a formação de um cidadão mais crítico e reflexivo, capaz de acompanhar as mudanças da sociedade e do mercado de trabalho de forma madura e diferenciada. ${ }^{4}$

No Brasil, foi justamente essa a intenção do governo federal ao criar o Programa Ciência sem Fronteiras, que promove consolidação, expansão e internacionalização da ciência e tecnologia, da inovação e competitividade brasileiras, por meio do intercâmbio e da mobilidade internacional durante 0 período universitário. ${ }^{5}$ Esse tipo de experiência agrega valor ao crescimento pessoal e profissional, pois viabiliza a vivência de diferentes hábitos e culturas, propicia momentos de construção da personalidade, desenvolvimento da autoconfiança, amadurecimento, independência e capacidade de relacionar-se. ${ }^{6}$

Particularmente na área da saúde, entre os vários temas pesquisados, a formação de profissionais tem sido objeto de preocupação constante das instituições de ensino superior, pois, por sua própria natureza, a universidade é produtora do conhecimento por meio da investigação. ${ }^{7}$ Dos futuros profissionais, têm sido exigidas, cada vez mais, distintas habilidades que transcendem o aprendizado técnico e incluem o domínio de outro idioma,
ISSN 2236 - 1987

conhecimento de diferentes culturas, competência para se relacionar, autoconfiança, desenvolvimento psicológico, amadurecimento e, sobretudo, capacidade de adaptação às mudanças impostas pelo mundo globalizado. ${ }^{5,8}$

Esse cenário evidencia a relevância da internacionalização do ensino superior, aqui concebida de forma ampliada, pois compreende, além da formação técnica, a inserção do estudante em uma dimensão internacional ou intercultural em todos os aspectos da educação e da pesquisa, o que é vital para o crescimento, tanto de países desenvolvidos, quanto para aqueles em desenvolvimento. ${ }^{9}$

Diante do exposto, a internacionalização dos cursos de graduação na área da saúde, e mais especificamente da Enfermagem, por meio do intercâmbio educacional internacional, pode contribuir de maneira significativa para a formação profissional desejada, considerando não apenas o avanço tecnológico a ser aprendido e aperfeiçoado, mas também, necessidade de estabelecer relações interpessoais saudáveis no atendimento ao cliente e à equipe profissional e, ainda, o enfrentamento das mudanças sociais e do mercado de trabalho.

Vale destacar também, que a temática em pauta ainda é insipiente nos estudos da área da Enfermagem, o que justifica a necessidade de buscar, na literatura, referências que possam facilitar o seu entendimento. Para tanto, construiu-se a seguinte questão norteadora: quais as características e temáticas abordadas nas publicações 
da área da saúde sobre intercâmbio educacional internacional vivenciados por estudantes de Enfermagem?

Assim, 0 presente estudo tem como objetivo analisar a produção do conhecimento científico a respeito de intercâmbios educacionais internacionais realizados por estudantes durante a graduação em Enfermagem.

\section{MATERIAIS E MÉTODOS}

Com a finalidade de alcançar o objetivo proposto, optou-se pelo método de revisão integrativa, visto que também permite reunir e sintetizar, de maneira organizada e sistemática, resultados de pesquisa sobre determinada temática. Essa opção contribui para o aprofundamento do tema investigado, além de indicar lacunas do conhecimento que precisam ser preenchidas com novas investigações. ${ }^{10}$

Para a realização da pesquisa, adotou-se os seguintes passos: escolha do tema e das palavras-chave; definição das bases de dados para busca; estabelecimento dos critérios para seleção da amostra; identificação do panorama geral do resultado da busca; elaboração de um formulário para registro dos dados coletados; análise e interpretação dos resultados. ${ }^{11}$

A coleta dos dados foi realizada no período de janeiro a junho de 2016, utilizando-se três bases de dados: Literatura Latino-Americana e do Caribe em Ciências da Saúde (LILACS), Medical Literature Analysis and
Retrieval System On-line (MEDLINE) e Biblioteca Cochrane (COCHRANE).

Para 0 levantamento bibliográfico, adotou-se como estratégia de busca a combinação dos seguintes descritores nos idiomas português, inglês e espanhol: intercâmbio AND internacional AND enfermagem. A fim de adotar uma terminologia comum para a localização de artigos científicos, a definição dos descritores teve como base os Descritores em Ciências da Saúde (DeCS), o que proporcionou uma forma padronizada e fidedigna para recuperação de informações, independentemente do idioma.

Constituíram critérios para a seleção da amostra: artigos publicados nos idiomas inglês, português e espanhol; artigos publicados no período compreendido entre 1995 e 2015 nos referidos bancos de dados e que abordassem os intercâmbios educacionais internacionais ocorridos durante o curso de graduação em Enfermagem na forma presencial. Quanto ao tipo de estudo, convém informar que além de estudos originais, integraram à pesquisa estudos reflexivos e de revisão de literatura, por se tratar de uma temática pouco abordada.

Excluíram-se estudos primários que versavam sobre intercâmbios realizados durante a pós-graduação, assim como experiências de intercâmbios por teleconferências, via web ou sob outra forma não presencial.

Após identificação dos artigos, realizou-se seleção de acordo com a questão norteadora e os critérios de inclusão previamente definidos. Todos 


\section{ISSN $2236-1987$}

os estudos identificados foram inicialmente avaliados por meio da análise de seus títulos e resumos. Nos casos em que esses não se mostraram suficientes para definir a seleção inicial, procedeu-se à leitura integral da publicação. De posse de todas as cópias dos artigos, realizou-se a leitura integral e crítica das publicações, para conferir se atendiam aos critérios de inclusão adotados no presente estudo.

Cabe informar que a busca foi replicada por dois pesquisadores com o intuito de garantir a aplicação correta dos procedimentos de busca e critérios de inclusão.

Figura 1 mostra o diagrama de fluxo da pesquisa:

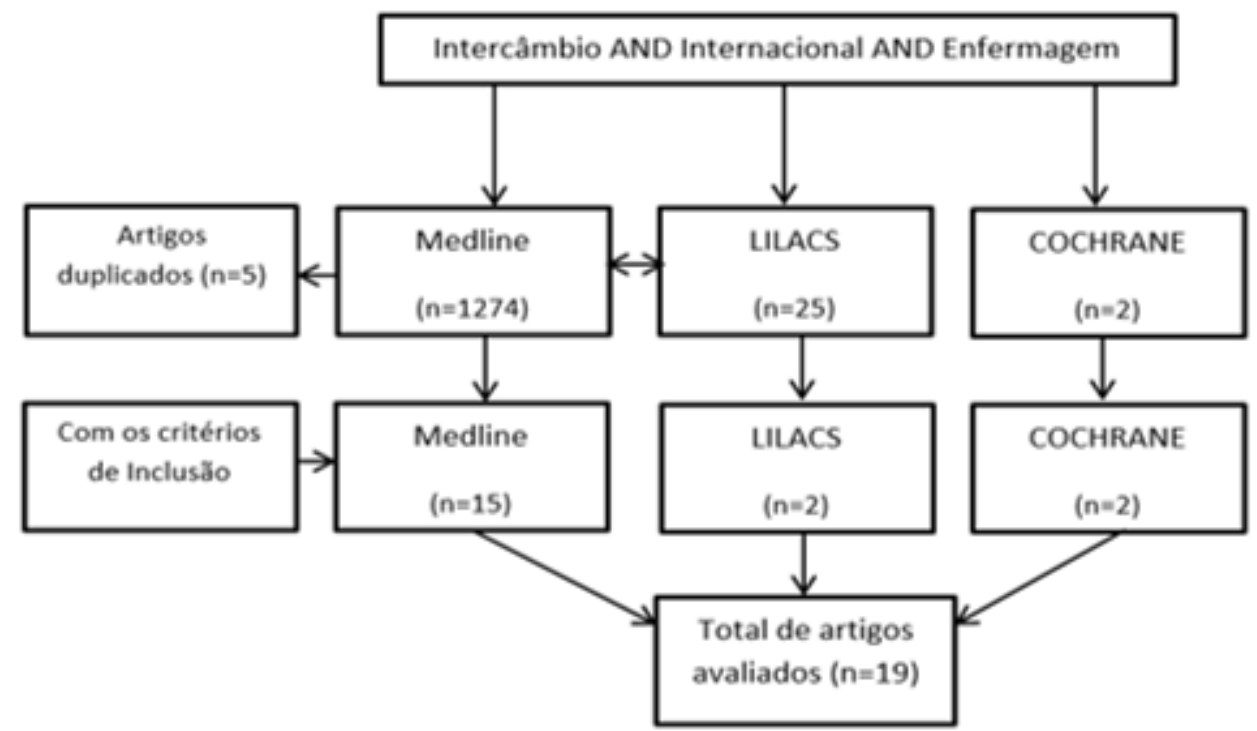

Figura 1 - Diagrama de fluxo do quantitativo dos artigos incluídos na revisão integrativa.

Para facilitar a coleta de dados, desenvolveu-se um instrumento de leitura, preenchido para cada artigo da amostra final do estudo, contendo itens como título e autores, ano e país de origem da publicação, delineamento metodológico, objetivos, principais resultados e conclusões. A utilização de tal instrumento permitiu a sucinta organização dos dados e facilitou a comparação dos estudos em tópicos específicos. Os resultados extraídos foram analisados de forma descritiva, discutidos à luz da literatura sobre o tema.

\section{RESULTADOS}

Compuseram o presente estudo 19 artigos $^{12-30}$ científicos, selecionados segundo os critérios previamente descritos. Ao analisá-los, identificouse a maioria na base de dados MEDLINE, com 80\% (15 artigos) das publicações, seguida pelas bases de dados LILACS e COCHRANE, com $11 \%$ (dois artigos) cada.

Em relação às datas de publicação, notou-se que os artigos foram publicados a partir de 2000, sendo a maioria entre 2012 e 2013, com 22\% (quatro artigos) em cada, 


\section{NURSING \\ AND HEALTH}

ISSN 2236 - 1987

seguidos de 2011, 2009 e 2006, com $11 \%$ (dois artigos) para cada um deles e um artigo publicado no ano de 2015.

Ao analisar os países de origem das publicações, verificou-se que
Estados Unidos da América (34\%) e Austrália (27\%) foram os mais frequentes, seguidos do Brasil e Reino Unido, ambos com 17\%. Esses dados estão ilustrados na Figura 2.

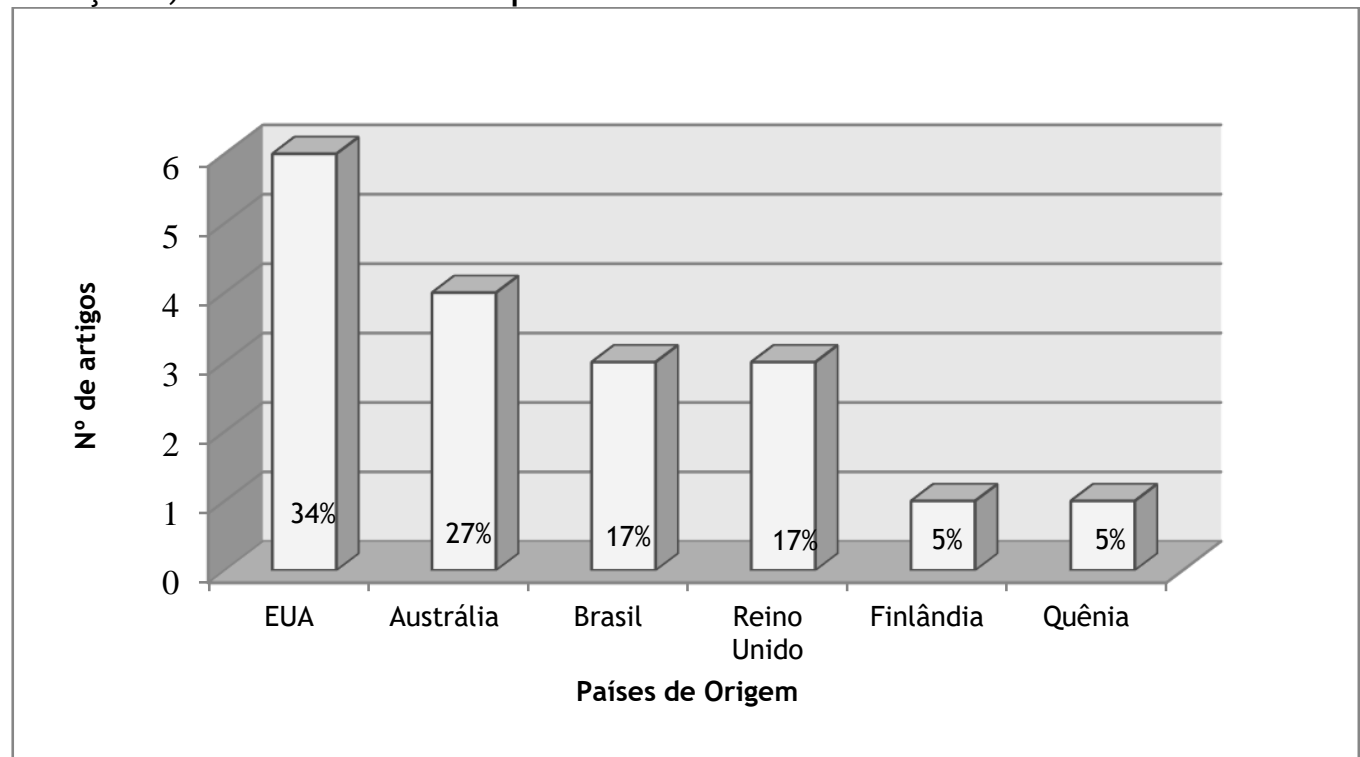

Figura 2 - Distribuição dos artigos pesquisados segundo o país de origem da publicação.

Quanto ao delineamento metodológico dos estudos pesquisados, verificou-se predomínio de pesquisas qualitativas em $42 \%$ dos estudos, seguido de relatos de experiência (31\%), estudos de revisão (21\%) e apenas um estudo (5,2\%) com abordagem quantitativa.

\section{DISCUSSÃO}

Os artigos selecionados sobre intercâmbios educacionais internacionais referem-se, em sua maior parte, àqueles realizados com os Estados Unidos e a Austrália, o que se atribui ao fato de serem países desenvolvidos, nos quais os investimentos na formação de pesquisadores são mais expressivos e, também, por serem considerados portas de entrada para muitos imigrantes e estudantes estrangeiros, fatos que os tornam países multiculturais. ${ }^{12}$

No que tange ao delineamento metodológico dos estudos analisados, verifica-se maioria de natureza qualitativa, na modalidade relato de experiência, possibilitando a expressão de muitas facetas pertinentes à esfera da subjetividade.

Permite visualizar o invisível no visível, mediante a percepção do outro. ${ }^{13}$ Portanto, é compreensível que estudos sobre 0 intercâmbio educacional internacional, na literatura estudada, estejam sendo conduzidos segundo o referencial da pesquisa qualitativa, em virtude de sua 
 \\ NOHEALTH \\ estreita relação com aspectos da experiência vivenciada, o que envolve apreensão de subjetividades.}

Quanto ao período de início das publicações analisadas, há convergência com a propagação e expansão do fenômeno da globalização, cujos efeitos tornaramse expressivos a partir de meados da década de 1990. Assim, aspectos considerados básicos para a ocorrência deste fenômeno estão interligados à temática pesquisada no presente estudo, a saber: comércio, transações financeiras e investimentos, migração e disseminação do conhecimento. ${ }^{14}$

Com base nos principais resultados e conclusões dos estudos analisados, evidenciaram-se aspectos similares relativos ao intercâmbio educacional internacional, entre os quais se destacaram quatro importantes temáticas: Desenvolvimento profissional e pessoal dos estudantes; Desafios e barreiras vivenciados no intercâmbio; Atividades realizadas durante o intercâmbio e Ensino interdisciplinar.

\section{Desenvolvimento profissional e pessoal dos estudantes}

A evolução profissional depende de aspectos contextuais de natureza social e institucional, o que a torna indissociável da estrutura pessoal e dos fatores que, direta ou indiretamente, interpenetram as relações interpessoais dos indivíduos. Este dimensionamento permite construir as perspectivas sobre a profissão e a própria identidade profissional. ${ }^{15}$

Segundo os relatos de experiência analisados neste estudo, o intercâmbio
ISSN 2236 - 1987

proporcionou uma experiência ímpar para o crescimento profissional e pessoal, pois oportunizou conhecer outro sistema de saúde e vivenciar a prática de enfermeiros da região, o que favoreceu a comparação com o modelo vigente no país de origem, contribuindo para a construção do pensamento crítico e maior valorização e respeito por sua própria cultura. A relação com professores e demais alunos constituiu outra justificativa, por favorecer o conhecimento de distintos contextos e sistemas, assim como 0 desenvolvimento de habilidades didáticas, pedagógicas e interpessoais. ${ }^{4,16-17}$

$\begin{array}{cc}\text { Ainda } & \text { considerando } \\ \text { desenvolvimento } & \text { profissional }\end{array}$
pessoal, estudo quantitativo realizado no Reino Unido, cujo objetivo foi avaliar $o$ impacto da experiência internacional dos estudantes de Enfermagem, evidenciou impacto positivo e significativo neste aspecto, na medida em que foram observadas transformações de atitudes e comportamentos dos estudantes após a experiência de intercâmbio. ${ }^{18}$ Eles mostraram-se mais maduros e com melhores habilidades de comunicação e tomada de decisões durante a assistência de Enfermagem? ${ }^{19-20}$

0 programa de intercâmbio permite que estudantes de enfermagem possam aprimorar suas habilidades de comunicação e atendimento aos usuários do sistema de saúde do país anfitrião, assim como possibilitar a compreensão de novos valores e culturas, também potencializando suas competências na comunicação e habilidades técnicas em distintas situações. ${ }^{21}$ 


\section{JOURNALOF \\ NURSING \\ ANO HEALTH}

ISSN 2236 - 1987

pois além de impactar na comunicação, interfere na interação social e na compreensão cultural. ${ }^{24}$ Nesse sentido, alguns autores ${ }^{25}$ sugerem estratégias para superar os desafios da internacionalização na área da saúde, uma delas relacionada ao idioma estrangeiro. Destaca-se que o ensino de uma língua estrangeira deve ser compulsório no ensino básico dos estudantes e defende-se a inserção de tal estudo nos cursos de graduação em Enfermagem, além do aperfeiçoamento da comunicação entre enfermeiros de outros países por meio das redes sociais. Os mesmos autores enfatizam ainda que a falta de domínio de um segundo idioma por parte dos estudantes tem dificultado uma inserção mais proativa nos países que recebem intercambistas.

Já no que se refere ao choque cultural, outro estudo norte-americano destacou essa dificuldade vivenciada pelos estudantes e descreveu alguns de seus sinais: euforia inicial, muitas vezes mascarando a ansiedade pela nova experiência; irritabilidade e desilusão que emergem ao redor da segunda semana, quando começam a perceber as diferenças no sistema de saúde; ajustamento ao processo de adaptação; e o estágio final, ou seja, a apreciação das diferenças e similaridades, momento em que os alunos desenvolvem habilidades no sentido de identificar as vantagens e desvantagens do sistema de saúde de ambos os países. Apesar dos níveis de estressores estarem presentes, a adaptação ocorre, trazendo benefícios ao processo de ensino e aprendizagem dos estudantes. ${ }^{26}$ idioma é o principal desafio que os estudantes e professores enfrentam, 


\section{JOURNALOF \\ NURSING \\ AND HEALTH}

ISSN 2236 - 1987

tutor-aluno entre professores de Enfermagem finlandeses e estudantes de intercâmbio britânico sob a perspectiva dos tutores. Este estudo concluiu que foram as relações de cuidado com os estudantes e não apenas as relações acadêmicas que fizeram diferença para minimizar o impacto do estresse cultural. 0 aspecto pastoral da relação foi essencial para auxiliar os alunos no esforço de estudar em um país estrangeiro. Por outro lado, os tutores foram incapazes de fornecerem o suporte necessário a todos para que superassem o choque cultural.

\section{Atividades desenvolvidas no} intercâmbio

Os artigos analisados relataram também algumas atividades desenvolvidas pelos estudantes durante 0 intercâmbio educacional internacional. Interessante observar que, apesar de estarem inseridos em um sistema de saúde e educacional, muitas vezes, bastante diverso em relação ao seu país de origem, alguns conseguiram vivenciar estágios em hospitais da região, realizar consultas de enfermagem, atividades de educação em saúde em comunidades rurais, ter envolvimento efetivo na equipe multidisciplinar, participar de visitas técnicas em hospitais regionais, bem como de grupos de pesquisas, e apresentar trabalhos científicos em eventos como congressos e seminários.

0 envolvimento dos estudantes, em especial, foi destacado em três estudos nacionais ${ }^{4,16-17}$ que relataram a oportunidade de realização de estágios curriculares e extracurriculares em hospitais da região, em diversos 


\section{NURSING \\ AND}

ISSN 2236 - 1987

de Enfermagem que experenciaram a prática clínica em comunidades, orfanatos, escolas e hospitais na Tailândia, evidenciou que a experiência transcende o desenvolvimento cultural, pois permite a valorização do trabalho em equipe. ${ }^{30}$

Nesse contexto, a educação interprofissional parece ser necessária e efetiva para o desenvolvimento de competências colaborativas, habilidades para o trabalho em equipe, compreensão dos papeis de outros profissionais e promoção de mudanças organizacionais. ${ }^{31}$ Esse trabalho colaborativo interdisciplinar entre outras categorias profissionais proporcionado pelos intercâmbios educacionais internacionais favorece também o desenvolvimento da saúde global que tem como prioridades a melhoria da saúde e o alcance da equidade na saúde para todos.

Os estudos indicam que o ensino interdisciplinar vivenciado pelos estudantes durante 0 intercâmbio favoreceu a construção do saber-fazer de outras categorias profissionais, levando ao melhor entendimento de suas limitações e identidade profissional.

\section{CONSIDERAÇÕES FINAIS}

A maioria dos estudos publicados era de natureza qualitativa, publicados nos Estados Unidos da América, Austrália e Brasil. Mediante análise, emergiram quatro temáticas: desenvolvimento profissional e pessoal, desafios e barreiras, atividades realizadas no intercâmbio e interdisciplinaridade e trabalho colaborativo. Essas temáticas 
permitiram explicitar experiências, dificuldades e benefícios potencialmente úteis aos interessados em intercâmbios desta natureza, a saber: alunos, Comissões de Graduação e outras esferas acadêmicas.

Também, reafirma a necessidade de novas pesquisas, conduzidas segundo abordagens metodológicas quantitativas, visto que a maioria dos estudos encontrados se norteou pela modalidade de pesquisa qualitativa e, particularmente, relatos de experiência, os quais prevaleceram nos estudos nacionais. Portanto, estudos brasileiros carecem de pesquisas de campo sobre a temática em questão.

Outro ponto observado diz respeito às estratégias utilizadas para minimizar os efeitos negativos decorrentes de intercâmbios. Diversos autores pontuaram a necessidade de estratégias, mas não as sugeriram efetivamente. Dessa forma, ressaltamos a carência de investigações que contemplem maior amostragem, conduzidas segundo distintas metodologias, para melhor avaliar tal impacto e contribuir para a perspectiva da empregabilidade do profissional enfermeiro.

Em relação às limitações, o presente estudo apresenta algumas: a utilização de pequeno número de base de dados (três) torna importante o desenvolvimento de nova pesquisa, abrangendo outras fontes, incluindo teses/dissertações, bibliotecas virtuais e até mesmo em buscadores acadêmicos. Relevante citar ainda o reduzido número de trabalhos nacionais (três) na modalidade relato de experiência, o que pode estar relacionado ao fato da pesquisa ter se reportado apenas aos cursos de Enfermagem, reafirmando a recomendação de busca mais ampla, englobando outros cursos de graduação.

Concluímos que, apesar dos intercâmbios educacionais internacionais no campo da Enfermagem ainda serem incipientes, trata-se de um fenômeno em expansão por estar atrelado aos efeitos do mundo globalizado. Assim, reafirma-se a importância de maiores investimentos governamentais e privados nos programas não só da pósgraduação, mas também, no âmbito da graduação.

\section{REFERÊNCIAS}

1. Celano AC, Guedes AL. Impactos da globalização no processo de internacionalização dos programas de educação em gestão. Cadernos EBAPE.BR. 2014;12(1):45-61.

2. Stromquist NP. Educação latinoamericana em tempos globalizados. Sociologias. 2012;14(29):72-99.

3. Klijn TP. Enfermería y globalización. Cienc enferm. 2010;16(1):9-15.

4. Oliveira MG, Pagliuca LMF. Programa de mobilidade acadêmica internacional em enfermagem: relato de experiência. Rev gauch enferm. 2012;33(1):195-8.

5. Bubadué RM, Carnevale F, Paula CC, Padoin SMM, Neves ET. Participação em programa de intercâmbio internacional: contribuições da experiência de graduação-sanduíche em enfermagem. Rev enferm UFSM 


\section{ISSN 2236 - 1987}

[Internet]. 2013 [acesso em 2016 ago 24]; 3(3):555-62. Disponível em: https://periodicos.ufsm.br/reufsm/ar ticle/view/7922/pdf

6. Santos WA, Leite BS, Valente GSC. 0 intercâmbio acadêmico internacional como estratégia educativa cultural: relato de experiência. Revista enfermagem profissional. 2014;1(2):304-14.

7. Marziale MHP. The regional transnationalization of Latin American nursing. Rev latino-am enfermagem. 2009;17(1):3-4.

8. Souza KV. Intercâmbio educacional internacional na modalidade doutorado sanduiche em enfermagem: relato de experiência. Esc Anna Nery. 2008;12(2):358-63.

9. Castro AA, Cabral Neto A. O ensino superior: a mobilidade estudantil como estratégia de internacionalização na América Latina. Revista lusófona de educação. 2012;21(21):69-96.

10. Mendes KDS, Silveira RCCP, Galvão CM. Revisão integrativa: método de pesquisa para a incorporação de evidências na saúde e na enfermagem. Texto contexto enferm. 2008;17(4):758-64.

11. Beyea SC, Nicoll ELH. Writing integrative review. Aorn j. 1998;67(4):877-80.

12. Spears E. O valor de um intercâmbio: mobilidade estudantil brasileira, bilateralismo \& internacionalização da educação. Rev eletrônica educ [Internet]. 2014 [ acesso em 2017 jun 20];8(1): 151-63. Disponível em: http://www.reveduc.ufscar.br/index. $\mathrm{php} /$ reveduc/article/view/1026
13. Lacerda MR, Labronici LM. Papel social e paradigmas da pesquisa qualitativa de enfermagem. Rev bras enferm. 2011;64(2):359-64.

14. Sousa ANL. Globalização: origem e evolução. Caderno de estudos ciência e empresa. 2011;8(1):2-16.

15. Mourão L, Porto JB, PuentePalacios K. Construção e evidências de validade de duas escalas de percepção de desenvolvimento profissional. Psico USF. 2014; 19(1):73-85.

16. Dantas RA, Pagliuca LMF. Experiência de intercâmbio em Portugal: uma pesquisa para a pesquisa. Rev RENE. 2004;5(1):101-6.

17. Dalmolin IS, Pereira ER, Silva RMCRA, Gouveia MJB, Sardinheiro JJ. Intercâmbio acadêmico internacional cultural: uma experiência de crescimento pessoal e científico. Rev bras enferm. 2013;66(3):442-7.

18. Thompson K, Boore J, Deeny P. A comparison of an international experience for nursing students in developed and developing countries. Int j nurs stud. 2000;37:481-92.

19. Mwenda AS. A Student's analysis of the moi university - linköping university exchange programme. Educ health. 2012;25(2):111-5.

20. Baernholdt M, Drake E, Maron F, Neymark K. Fostering internationalization: an AmericanDanish semester-long undergraduate nursing student exchange program. Int nurs rev. 2013;60(2):221-7.

21. Wood MJ, Atkins M. Immersion in another culture: one strategy for increasing cultural competency. J cult divers. 2006;13(1):50-4. 


\section{NURSING \\ AND HEALTH}

ISSN 2236 - 1987

22. Callister LC, Cox AH. Opening our hearts and minds: the meaning of international clinical nursing electives in the personal and professional lives of nurses. Nurs health sci. 2006;8(2):95-102.

23. Kelleher S. Perceived benefits of study abroad programs for nursing students: an integrative review. J nurs educ._2013;52(12):690-5.

24. Wang CC, Andre K, Greenwood KM. Chinese students studying at Australian universities with specific reference to nursing students: a narrative literature review. Nurse educ today. 2015;35(4):609-19.

25. Backes DS, Backes MS, Lunardi VL, Erdmann AL, Buscher A. Internationalization as a challenge to the impact of globalization: nursing contributions. Rev esc enferm USP. 2014;48(5):772-7.

26. Egenes KJ. Health care delivery through a different lens: The lived experience of culture shock while participating in an international educational program. Nurse educ today. 2012;32(6):760-4.

27. Smith RA, Khawaja NG. A review of the acculturation experiences of international students. Int $\mathrm{j}$ intercult relat. 2011;35(5):699-713.

28. Edgecombe $K$, Jennings $M$, Bowden $M$. International nursing students and what impacts their clinical learning: literature review. Nurse educ today. 2013;33(2):138-42.

29. Koskinen L, Tossavainen $\mathrm{K}$. Relationships with undergraduate nursing exchange students - a tutor perspective. $\mathrm{J}$ adv nurs. 2003;41(5):499-508.
30. Reid-Searl K, Dwyer T, Moxham L, Happell B, Sander T. Rediscovering the essence of nursing: Exploring the impact of in clinical experience in Thailand for undergraduate nursing students from Australia. Nurse educ today. 2011;31(8):892-7.

31. Curran V, Sargeant J, Hollett A. Evaluation of an interprofessional continuing professional development initiative in primary health care. J contin educ health prof. 2007;27(4):241-52.

Data de submissão: 14/04/2017

Data de aceite: 10/08/2017

Data de publicação: 30/08/2017 